\title{
The Role of Grain Boundary Sliding in Microstructural Evolution during Superplastic Deformation of a 7055 Aluminum Alloy
}

\author{
Rustam Kaibyshev ${ }^{1}$, Alexandre Goloborodko ${ }^{2}$, Fanil Musin ${ }^{1}$, Ilya Nikulin ${ }^{1}$ and Taku Sakai ${ }^{2}$ \\ ${ }^{1}$ Institute for Metals Superplasticity Problems, Khalturina 39, Ufa 450001, Russia \\ ${ }^{2}$ Department of Mechanical and Control Engineering, The University of Electro-Communications, Chofu 182-8585, Japan
}

\begin{abstract}
The microstructure evolution in a 7055 aluminum alloy subjected to thermomechanical processing (TMP) was studied at $450^{\circ} \mathrm{C}$ and $\dot{\varepsilon}=1.7 \times 10^{-3} \mathrm{~s}^{-1}$ at which the material exhibits superplastic behavior with a total elongation of $720 \%$ and the coefficient $m=0.58$. Partially recrystallized initial structure of the as-processed $7055 \mathrm{Al}$ consisted of bands of recrystallized grains with a mean size of $11 \mu \mathrm{m}$ alternating with bands of recovered subgrains with a mean size of $2 \mu \mathrm{m}$. The true stress-true strain curve exhibits a well-defined peak stress, followed by gradual strain softening. The coefficient of strain rate sensitivity, $m$, remains unchanged at $\varepsilon \leq 1$ and tends to decrease with strain at $\varepsilon>1$. The initial microstructure persists near the peak strain. Following strain leads to evolution of initial partially recrystallized structure into uniform fully recrystallized structure due to occurrence of continuous dynamic reactions, i.e. continuous dynamic recrystallization (CDRX). The data of microstructural observation and misorientation analysis show that low-angle boundaries (LAB) gradually convert to high-angle boundaries $(\mathrm{HAB})$ resulting in an extensive flow softening. It was shown that grain boundary sliding (GBS) provides superplastic flow at all strains. Concurrently, GBS plays an important role in the dynamic evolution of new grains facilitating conversion of LABs to HABs.
\end{abstract}

(Received May 2, 2002; Accepted June 11, 2002)

Keywords: superplastic deformation, aluminum alloys, microstructure evolution, grain boundary sliding, electron back-scattering diffraction (EBSD) analysis, continuous dynamic recrystallization

\section{Introduction}

Aluminum alloys are capable of superplastic deformation in two distinctly different microstructural conditions. First, aluminum alloys with a fully recrystallized structure with a mean grain size less than $10 \mu \mathrm{m}$ exhibit superplastic behavior. ${ }^{1,2)}$ The origin of superplasticity in aluminum alloys with such the structure is enough clear. ${ }^{2)}$ At higher temperatures, the grain boundary sliding (GBS) occurs through the relative displacement of fine grains providing superplastic flow. ${ }^{1,2)}$ The fine grained structure is produced by twostep thermomechanical processings (TMP). There are two different TMP routes to achieve grain refinement in wrought aluminum alloys. One of these routes termed as overaging/recrystallization TMP, ${ }^{1,3-6)}$ involves overaging treatment to produce coarse precipitate particles, which become sites for particle-stimulated nucleation of static recrystallization after cold rolling. The other route, which can be termed as recrystallization/recrystallization TMP, ${ }^{7-9)}$ involves two sequential procedures of warm/cold rolling followed by recrystallization annealing.

Second, aluminum alloys with initial unrecrystallized structure consisting of cells or recovered subgrains and containing fine dispersoids as $\mathrm{Al}_{3} \mathrm{Zr}$ are capable of superplasticity. ${ }^{1,5,6)}$ These aluminum alloys are subjected to extensive cold or warm rolling followed by heating to temperatures of superplastic deformation. ${ }^{1,5,6,10-20)}$ Dispersion particles prevent static recrystallization of the warm/cold worked material, and plastic deformation induces high-angle boundaries (HAB) via a continuous reaction called as continuous dynamic recrystallization (CDRX). ${ }^{1,2-20)}$ The dispersoids effectively pin the deformation-induced boundaries preventing much of the low-angle boundary (LAB) migration resulting in their mutual elimination ${ }^{21)}$ and also growth of recrystallized grains as well that provides capability for superplasticity. De- spite considerable research works, the exact nature of such the recrystallization process and origin of superplasticity of aluminum alloys in this microstructure condition are not yet clear. $^{1,20)}$ It has been proposed ${ }^{12,17-20)}$ that LABs are evolved in cellular dislocation structure under static annealing preceded superplastic deformation. Following deformation results in the eventual conversion of these LABs into HABs, which become able to support GBS. In this manner the aluminum alloys achieve superplasticity during high temperature straining.

The present work was initiated with intent of examining the mechanism of superplasticity in the aluminum alloys in the second microstructural condition. It was recently shown that a 7055 aluminum alloy with a unique initial structure subjected to the two-step TMP exhibits superplasticity with a total elongation of $720 \%$ and the coefficient $m=0.58$ at $T=450^{\circ} \mathrm{C}$ and $\dot{\varepsilon}=1.7 \times 10^{-3} \mathrm{~s}^{-1} .^{22)}$ This alloy had a partially recrystallized structure consisted of bands of recrystallized grains with a mean size of $11 \mu \mathrm{m}$ alternating with bands of recovered subgrains with a mean size of $2 \mu \mathrm{m} .^{22)}$ This material was, therefore, capable for superplasticity in microstructure state being intermediate between two conditions described above. Analysis of microstructural evolution during superplastic deformation of this 7055 alloy allows elucidating the mechanism of CDRX and revealing the role of CDRX in the superplasticity.

A main goal of the present study is to characterize the evolution of microstructure in the $7055 \mathrm{Al}$ during superplastic deformation. It was recently suggested that GBS along HABs can result in rotation of subgrains near the HABs, leading to a rapid transformation of the subgrains into true recrystallized grains. ${ }^{13,15,23)}$ There are, however, limited research works provided direct evidence for operation of GBS in unrecrystallized structure. Thus, the second aim of this study is to reveal the role of GBS in microstructural evolution during superplastic deformation and to relate the microstructure 
evolved with mechanical response of the material.

\section{Experiments}

The material was the same 7055 aluminum alloy as in the previous study ${ }^{22}$ and the TMP applied to this material for grain refinement was already described in details elsewhere. ${ }^{22)}$ The final thickness of the sheet produced via this TMP route was $\sim 1.4 \mathrm{~mm}$. Tensile samples with a $10 \mathrm{~mm}$ gauge length and $4 \mathrm{~mm}$ width were machined directly from these sheets; the loading axis was parallel to the rolling direction. The specimens were deformed in tension to fixed true strains $0.4,0.7,1.1$, and 1.7 at $450^{\circ} \mathrm{C}$ and $\dot{\varepsilon}=1.7 \times 10^{-3} \mathrm{~s}^{-1}$. Details of the tension tests and optical metallography examinations were described elsewhere. ${ }^{22)}$

All structural studies were carried out on the longitudinal (tension) and long transverse sections of specimens. For EBSD analysis, the strained samples were mechanically polished and, finally, electropolished in the solution containing $10 \mathrm{ml}$ of perchloric acid and $90 \mathrm{ml}$ buthanol at $10^{\circ} \mathrm{C}$. Misorientations of (sub)grain boundaries were determined by EBSD technique by means of hard and software of TexSem Laboratories, Inc. (Provo, UT) installed on Hitachi-3500A SEM. For the surface observations the samples mechanically polished were covered by boric acid to prevent oxidation at high temperatures and then strained. The deformation relief of these samples was studied using a JSM-840 scanning electron microscope (SEM).

\section{Results}

\subsection{Initial microstructure}

The microstructure of the $7055 \mathrm{Al}$ developed after the two-step TMP (Fig. 1(a)) has been described in detail elsewhere. ${ }^{22}$ The data of EBSD examination of the initial microstructure is shown in Fig. 1(b). It is seen that the boundary misorientation distribution is distinctly bimodal, there is large fractions of LABs with misorientation less than $5 \mathrm{deg}$ $(\sim 30$ pct.) and HABs misoriented in the range 20 to $60 \mathrm{deg}$ ( 70 pct.).

\subsection{Mechanical properties}

A true stress-true strain curve for the 7055 aluminum alloy at $450^{\circ} \mathrm{C}$ and an initial strain rate of $1.7 \times 10^{-3} \mathrm{~s}^{-1}$ is presented in Fig. 2(a). It is seen that a well defined peak in flow stress appears at a peak strain, $\varepsilon_{\mathrm{p}}$, of $\sim 0.4$ following an extensive strain hardening, and then a gradual strain softening takes place in high strain. A stress oscillation of flow curves appears at $\varepsilon \geq 0.9$. Their amplitude tends to increase with strain. The coefficient of strain rate sensitivity, $m$, of 0.58 remains virtually unchanged at $\varepsilon \leq 1$ (Fig. 2(b)). Cyclic drops in the $m$ value are accompanied with the stress oscillations. Strain rate sensitivity decreases from 0.58 to 0.5 with increase in strain from 1 to 1.2 , and then from 0.5 to 0.4 in the strain interval 1.4-1.7. At $\varepsilon \geq 1.9$, the $m$ value decreases less than 0.33 which can suggest the termination of superplastic flow. ${ }^{4,5,9)}$
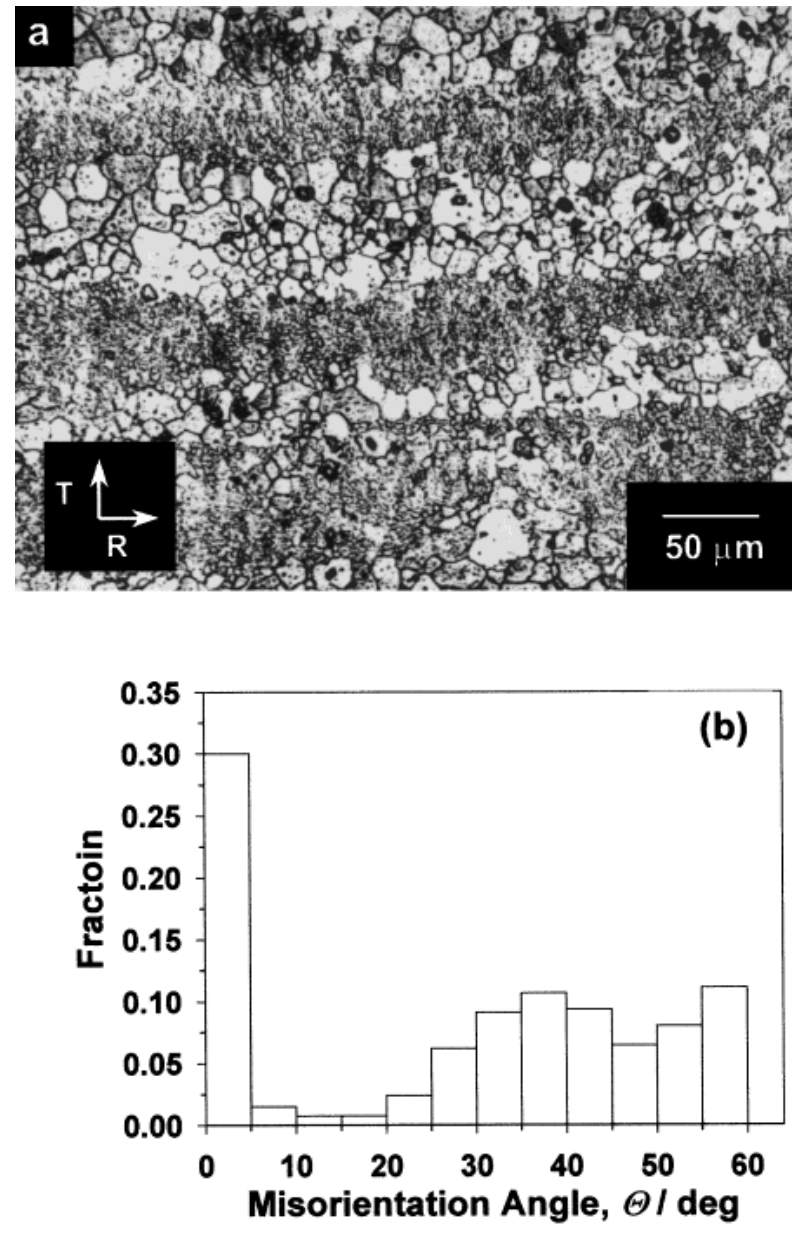

Fig. 1 Initial microstructure of the 7055 alloy subjected to the two-step TMP. (a) Optical microscopy and (b) misorientation diagram of (sub)grain boundaries.

\subsection{Microstructural evolution 3.3.1 Optical microscopy}

It was previously reported $^{22)}$ that static annealing of the $7055 \mathrm{Al}$ at $450^{\circ} \mathrm{C}$ led to a slight decrease in the volume fraction of the unrecrystallized regions and insignificant growth of recrystallized grains. There was almost no change in the crystallite size in the unrecrystallized regions. ${ }^{22)}$ Extensive structural changes occur, however, under superplastic deformation. Figure 3 shows microstructures of the 7055 Al deformed to strains of $0.4,0.7$ and 1.7 at $450^{\circ} \mathrm{C}$ and $\dot{\varepsilon}=1.7 \times 10^{-3} \mathrm{~s}^{-1}$. Microstructure evolved at strains of 1.1 and 2.1 were presented elsewhere. ${ }^{22)}$ The structural characteristics are summarized in Fig. 4. It is seen, that superplastic deformation results in gradual transformation of subgrains in the unrecrystallized regions to grains surrounded by HABs (Figs. 3 and 4) which can be evident from enhanced contrast in etched boundaries of crystallites. As a result, the volume fraction of unrecrystallized regions decreases with strain and almost fully recrystallized structure is developed at $\varepsilon \geq 1.1$ (Figs. 3(c) and 4). Transformation of subgrains into grains is accompanied by an extensive grain growth (Figs. 3 and 4). The growth of grains evolved in the unrecrystallized regions occurs much faster than that in the recrystallized regions (Fig. 3) because of the size of grains initially evolved in the unrecrystallized regions is $\sim 3$ times smaller than that of the recrystallized grains, and this size tends to approach 

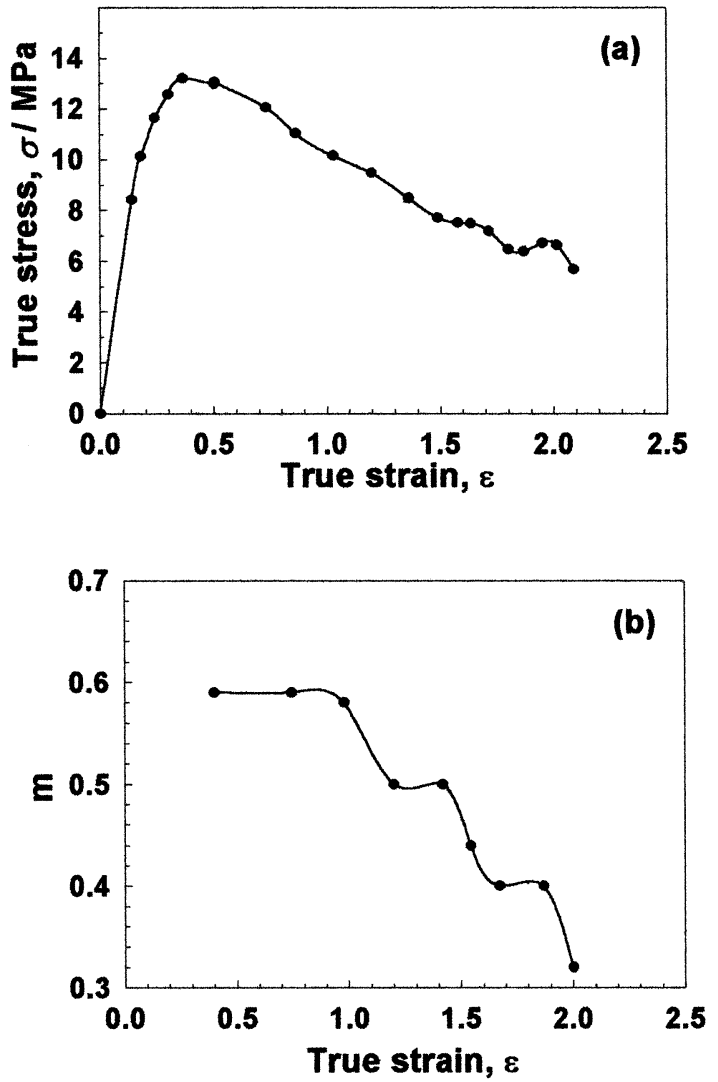

Fig. 2 Superplastic response of the $7055 \mathrm{Al}$ strained at $450^{\circ} \mathrm{C}$ and $\dot{\varepsilon}=1.7 \times 10^{-3} \mathrm{~s}^{-1}$. (a) True stress as a function of true strain and (b) effect of strain on the coefficient of strain rate sensitivity, $m$.

to an equilibrium value $(\sim 15 \mu \mathrm{m})$ for this temperature-strain rate conditions. ${ }^{1,24)}$ The grain aspect ratio, defined as the ratio of the grain dimension in the longitudinal direction to that in the transverse direction increases at $\varepsilon \leq 1.1$ and remains essentially constant with further straining.

\subsubsection{Boundary character data}

Figure 5 represents typical orientation image microscopy (OIM) maps for superplastically deformed specimens; Fig. 6 illustrates the distribution of misorientation for these samples. It is seen (Figs. 5 and 6) that superplastic deformation results in conversion of initial LABs into HABs in unrecrystallized areas. This is indicative from increased fraction of deformation-induced boundaries with misorientation ranging from 5 to $20 \mathrm{deg}$ (Fig. 6(a)). As a result, the formation of crystallites with a mean size of $\sim 5 \mu \mathrm{m}$ outlined by HABs is detected here as can be seen in Fig. 5(a). At $\varepsilon=0.4$, fine grains of $\sim 5 \mu \mathrm{m}$ alternate with initially recrystallized grains with a mean size of $\sim 15 \mu \mathrm{m}$ (Figs. 5 (a) and (b)). The recrystallized grains grow gradually with strain (Fig. 5(b)). Concurrently, the formation of new LABs occurs within recrystallized grains. This is evident from the increased fraction of LABs ( $\leq 5 \mathrm{deg}$ ) in regions of recrystallized grains (Fig. 6(b)). As a result, there exists remarkable fraction of LABs although most of boundaries in recrystallized areas exhibit high angle misorientation (Fig. 6(b)).

Further strain to 0.7 enhances uniformity of microstructure (Fig. 5(c)) although distinctly bimodal distribution of misorientations is observed again (Fig. 6(c)). Increased fraction of LABs is attributed to the formation of these boundaries in
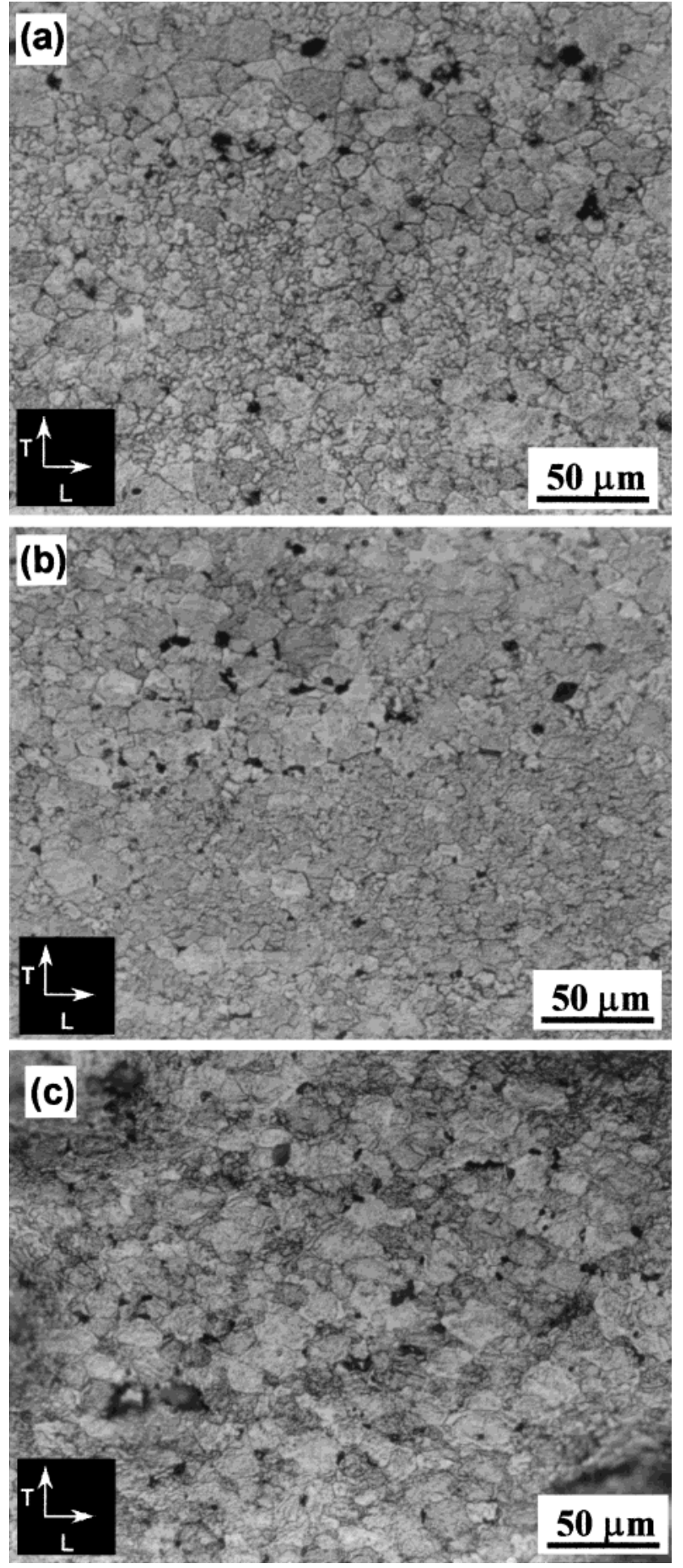

Fig. 3 Microstructures evolved during deformation at $T=450^{\circ} \mathrm{C}$ and at $\dot{\varepsilon}=1.7 \times 10^{-3} \mathrm{~s}^{-1}$. (a) $\varepsilon=0.4$, (b) $\varepsilon=0.7$ and (c) $\varepsilon=1.7$.

initially recrystallized grains (Fig. 5(c)). Most of the LABs exhibits misorientation of $\sim 2 \mathrm{deg}$. Following strain leads to increasing grain size (Figs. 5(d) and (e)); an extensive formation of LABs with misorientation less than 2 deg (Figs. 6(d) and (e)) occurs. It is worth noting that some boundaries outlining crystallites with size ranging from 6 to $12 \mu \mathrm{m}$ retain low-angle misorientation at $\varepsilon=1.1$ (Fig. 5(d)), and all such boundaries become HABs at $\varepsilon=1.7$ (Fig. 5(e)). 


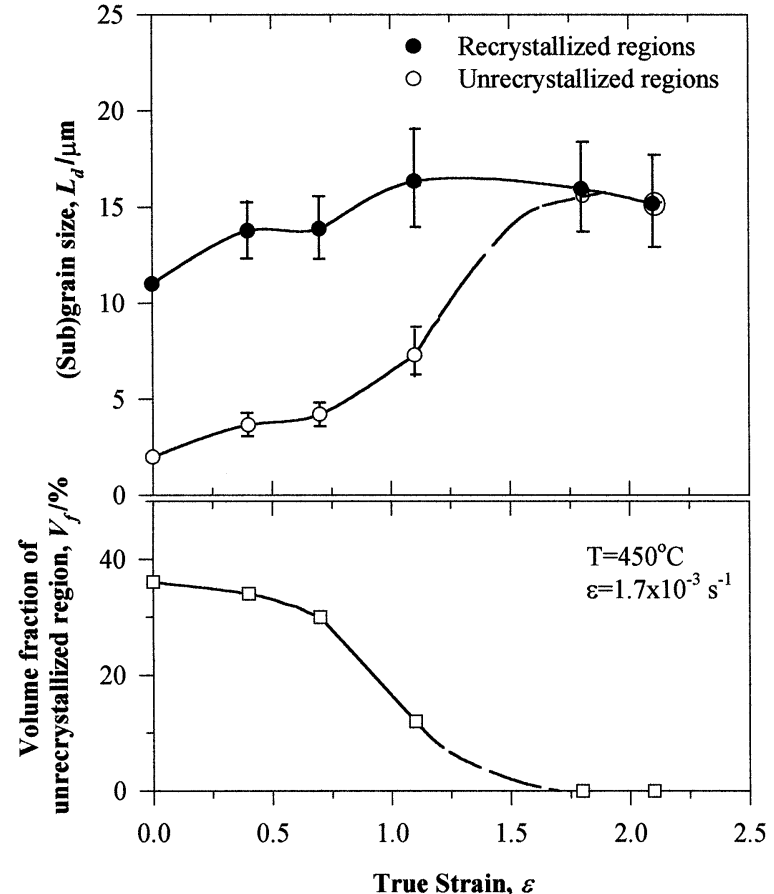

Fig. 4 Effect of strain on sizes of (sub)grain in unrecrystallized areas, $L_{\mathrm{sd}}$, grain size, $L_{\mathrm{d}}$, in recrystallized areas and volume fraction of unrecrystallized structure, $V_{\mathrm{f}}$, at $450^{\circ} \mathrm{C}$ and $\dot{\varepsilon}=1.7 \times 10^{-3} \mathrm{~s}^{-1}$.

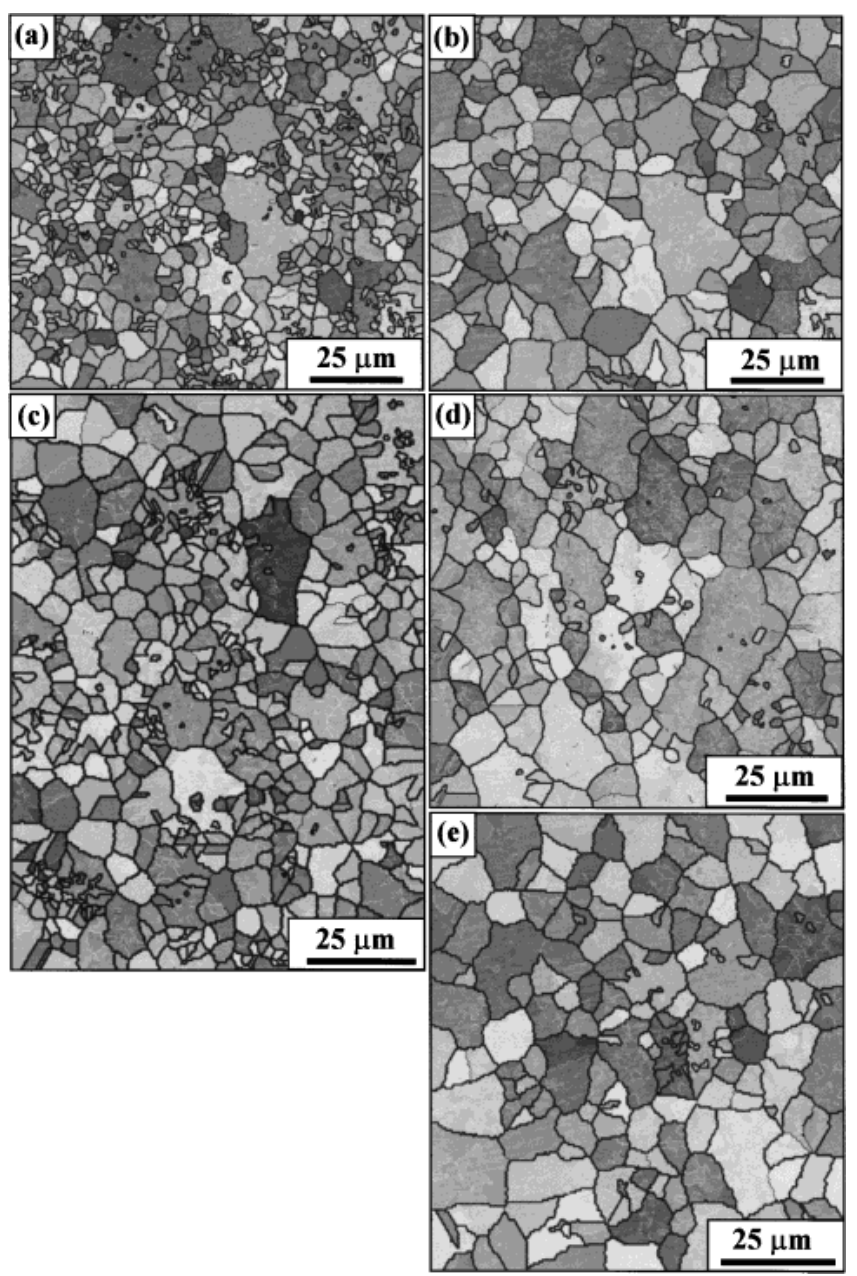

Fig. 5 Typical OIM maps of specimens strained to (a) $\varepsilon=0.4$, a typical unrecrystallized area; (b) $\varepsilon=0.4$, a typical recrystallized area, (c) $\varepsilon=0.7$, (d) $\varepsilon=1.1$ and (e) $\varepsilon=1.7$.
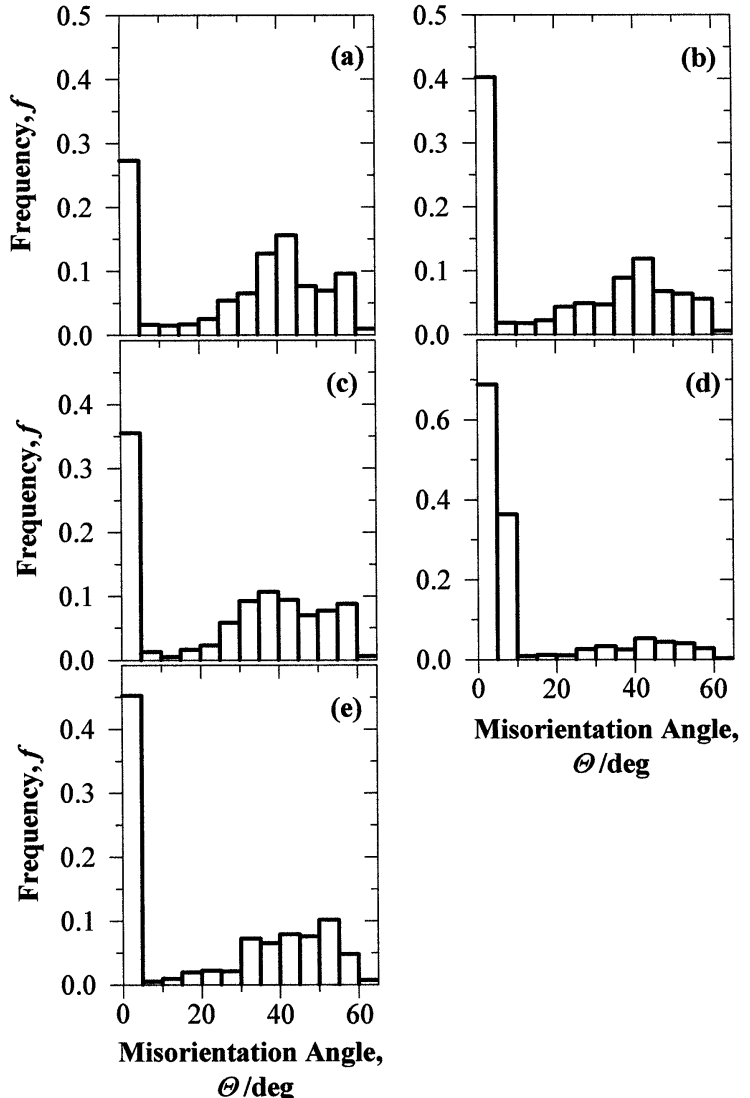

Fig. 6 (Sub)grain boundary misorientation histograms illustrating the distribution of misorientations in samples strained to (a) $\varepsilon=0.4$, a typical unrecrystallized area, (b) $\varepsilon=0.4$, a typical recrystallized area, (c) $\varepsilon=0.7$, (d) $\varepsilon=1.1$ and (e) $\varepsilon=1.7$.

\subsection{Surface observations}

At strains less than the peak strain, $\varepsilon_{\mathrm{p}}(\leq 0.4)$, the deformation relief is inhomogeneous. It can be seen in Fig. 7(a) that GBS occurs in a individual manner throughout the sliding of individual grains in recrystallized areas and, in contrast, along separate boundaries in unrecrystallized areas. There is an evidence for operation of GBS in a cooperative manner through the shift of grain groups as a unit relative to each other along common grain boundary surfaces ${ }^{25,26)}$ which propagate into the unrecrystallized areas. These surfaces locate at angles ranging from 60 to $85 \mathrm{deg}$ to the tension axis. At $\varepsilon \sim 0.4$, an extensive propagation of cooperative GBS takes place throughout the unrecrystallized areas (Fig. 7(b)). The size of the grain groups sliding as a unit is equal to a size of about 5 crystallites. At higher strain, operation of GBS takes place both in the individual manner and in the cooperative manner as well in unrecrystallized areas (Fig. 7(c)). The most of surfaces of cooperative GBS were found to be located at an angle of $\sim 45^{\circ}$ to the tension axis. At $\varepsilon>1.1$, uniform GBS via sliding of individual grains dominates (Fig. 7(d)). It can be evident that most of boundaries, along which GBS takes place, locate at an angle of $\sim 45^{\circ}$ to the tension axis. Thus, at low strain, GBS of individual grains begins to occur in recrystallized areas. Then with increasing strain the cooperative GBS occurs throughout unrecrystallized areas, followed by sliding of individual grains in whole material body at high strains. 

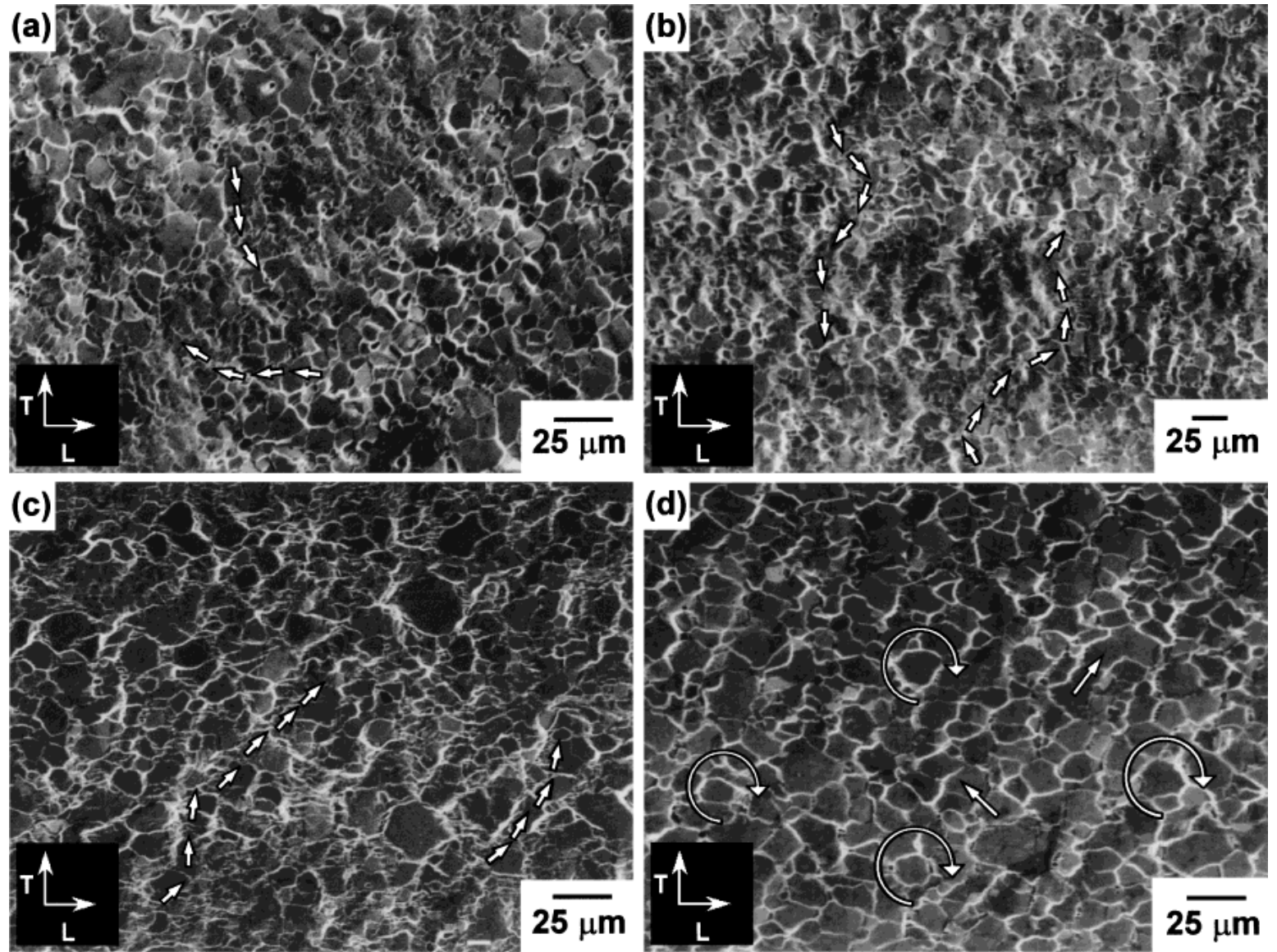

Fig. 7 Surface observations after superplastic deformation at $450^{\circ} \mathrm{C}$ and $\dot{\varepsilon}=1.7 \times 10^{-3} \mathrm{~s}^{-1}$. Arrows indicate propagation of GBS along common grain boundary surfaces. (a) $\varepsilon=0.2$, (b) $\varepsilon=0.4$, (c) the sample was strained to 0.7 then was unloaded, polished and then additionally strained to $\varepsilon=0.3$, i.e. the total strain was about 0.8 , (d) the sample was strained to 1.1 then was unloaded, polished and then additionally strained to $\varepsilon=0.3$, i.e. the total strain was about 1.2 .

\section{Discussion}

The results of present study described above indicate that superplastic response of the present $7055 \mathrm{Al}$ with initial partially recrystallized structure can be connected with dynamic evolution of new grains, i.e. CDRX. The experimental data suggest that GBS can play a very important role in the microstructure evolution. A model of the microstructure evolution during superplastic deformation (Fig. 8) must be able to explain the mechanical behavior of the $7055 \mathrm{Al}$ and observed changes in grain boundary characters.

At $\varepsilon<\varepsilon_{\mathrm{p}}$, GBS is supported by HABs outlining recrystallized grains (Fig. 8(a)). These boundaries locating at different angles to the tension axis and therefore occurrence of GBS requires increased shear stress since the Schmid factor for these boundaries is significantly less than that for boundaries locating at an angle of $\sim 45^{\circ}$ to the tension axis. ${ }^{25,26 \text { ) }}$ Concurrently, the easier shifting of grain groups in recrystallized areas as a unit begins to occur along common grain boundary surfaces (Fig. 8(a)). It is apparent that cooperative GBS propagates into unrecrystallized regions, and this propagation can occur, for instance, along prior existed separate HABs in unrecrystallized areas. Shift of grain groups as a unit in unrecrystallized areas results in rotation of neighboring subgrains (Fig. 8(b)), as it was established in the previous report. ${ }^{23)}$ It seems that propagation of GBS to unrecrystal- lized regions and the involving of almost all initially recrystallized grains in supporting GBS are provided by progressive increase in flow stress which attends a peak (Figs. 2, 8(a) and (b)). Cooperative GBS facilitates highly the transformation of LABs to HABs in unrecrystallized areas. This process occurs with a relatively high rate and, as a result, the sufficient number of HABs being capable of supporting GBS is evolved in unrecrystallized areas at $\varepsilon \sim \varepsilon_{\mathrm{p}}$.

At $\varepsilon>\varepsilon_{\mathrm{p}}$, the number of fine crystallites outlined by HABs from all sides increases and GBS starts to occur in the individual manner in unrecrystallized areas (Fig. 8(c)). At these strains, both sliding of individual grains and cooperative GBS begin to take place by easiest way along boundaries located at an angle of $\sim 45^{\circ}$ to the tension axis (Fig. 8(d)). Transition to dominant sliding in direction with favorable Schmid factor facilitates GBS providing strain softening. As a result, the flow stress gradually decreases with strain.

At higher strains, grain growth is the main process of microstructural evolution and the sliding of individual grains becomes the main deformation mechanism (Fig. 8(d)). New LABs are formed inside the grains. However, these boundaries transform to true HABs with a very low rate due to the fact that no GBS occurs along these LABs.

It is worth noting that the coefficient of strain sate sensitivity, $m$, remains unchanged at the stages of superplastic deformation when the number of boundaries supporting GBS 


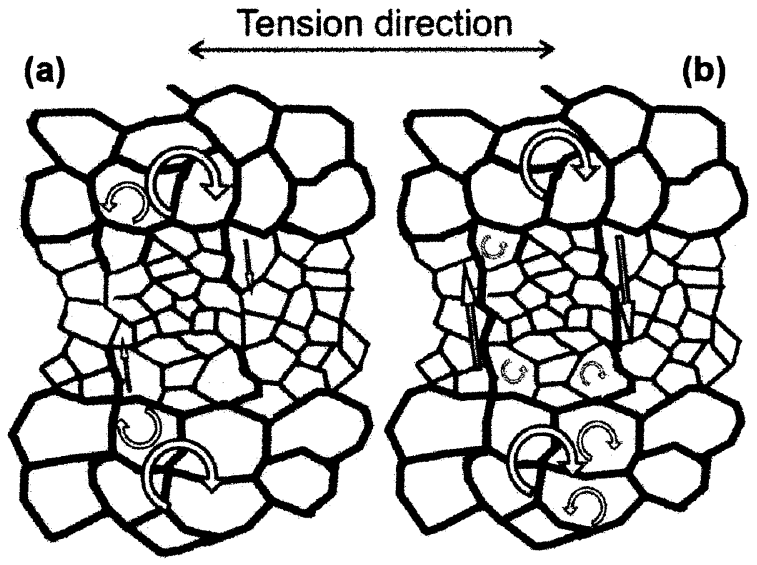

(c)

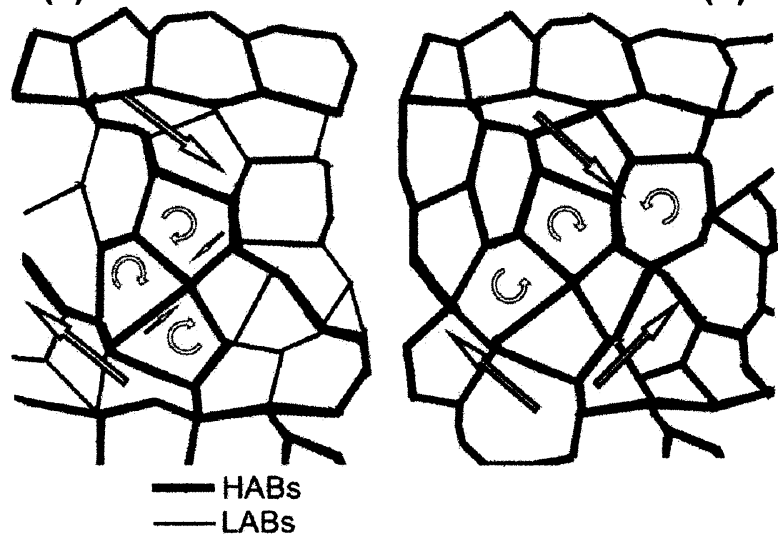

Fig. 8 Schematic representation of dynamic evolution of new grains correlating with mechanical response of the $7055 \mathrm{Al}$ at different strain. (a) $\varepsilon<\varepsilon_{\mathrm{p}}$, GBS operating in individual manner in recrystallized areas and propagation of cooperative GBS into unrecrystallized areas, (b) $\varepsilon \sim \varepsilon_{\mathrm{p}}$, initiation of subgrain rotation promoting transformation of LABs into HABs by cooperative GBS in unrecrystallized areas, (c) $\varepsilon>\varepsilon_{\mathrm{p}}$, development of cooperative GBS along common boundary surfaces locating at an angle of $\sim 45^{\circ}$ to the tension axis and operation of individual GBS in recrystallized areas, (d) high strain, dominant sliding of individual grains along boundaries locating at an angle of $\sim 45^{\circ}$ to the tension axis.

increases with strain $(\varepsilon \leq 1)$. The $m$ value tends to decrease at higher strains where grains become to grow extensively. Perhaps, it is associated with decreasing contribution of GBS and increasing contribution of dislocation glide into total deformation with strain at $\varepsilon>1$.

Thus, it can be concluded that superplastic behavior of aluminum alloys in the second microstructural condition is also attributed to GBS along HABs. At small stains, this GBS occurring along separate HABs induces rotation of subgrains providing facilitated transformation of LABs to HABs. CDRX resulting in uniform grained structure provides occurrence of GBS through the relative displacement of fine grains at high strains.

Transition of the operation of GBS from cooperative manner in low strain, to dominant sliding of individual grains in high strain yields unusual strain dependence of cavitation. ${ }^{22)}$ At low strains, a plasticity-controlled cavity growth, associated with hindered accommodation of cooperative GBS in the unrecrystallized areas, results in the formation of coarse cavities. $^{22)}$ At high strains, the easy accommodation of GBS occurring in the individual manner suppresses plasticity-controlled cavity growth. A diffusion-controlled cavity growth resulting in the formation of fine cavities dominates at high strain. As a result, strain increase leads to decreasing cavity size.

\section{Conclusion}

The microstructural evolution as well as superplastic response of the $7055 \mathrm{Al}$ alloy with a partially recrystallized structure was studied at $450^{\circ} \mathrm{C}$ and $\dot{\varepsilon}=1.7 \times 10^{-3} \mathrm{~s}^{-1}$. The $\sigma-\varepsilon$ curve shows a strain hardening and a well-defined peak stress at a relatively low strain, followed by a gradual strain softening. At the initial hardening stage, low-angle boundaries in the unrecrystallized regions start to convert to highangle boundaries and this process extensively occurs at the stage of strain softening resulting in the formation of fully recrystallized structure at $\varepsilon>1.1$. It is concluded that transformation of low-angle boundaries to high-angle boundaries can be highly promoted by operation of grain boundary sliding. Peak stress can be associated with initiation of GBS in unrecrystallized regions of recovered subgrains. Strain softening is associated with increasing number of HABs with strain. The sliding of fine individual grains occurs mostly along boundaries locating at an angle of $\sim 45^{\circ}$ to the tension axis at $\varepsilon \geq 1.1$.

\section{Acknowledgments}

This work was supported in part by the International Science and Technology Center under Project no. 2011. One of the authors (A.G.) wishes to thank to the Japanese Government for providing the scholarship.

\section{REFERENCES}

1) F. J. Humphreys and M. Hatherly: Recrystallization and Related Annealing Phenomena, (Oxford: Pergamon Press, 1996) p. 414.

2) J. A. Wert, N. E. Paton, C. H. Hamilton and M. W. Mahoney: Metall. Trans. 12A (1981) 1267-1276.

3) L. P. Troeger and E. A. Starke, Jr.: Mater. Sci. Eng. A277 (2000) 102 113.

4) T. G. Nieh, J. Wadsworth and O. D. Sherby: Superplasticity in Metals and Ceramics, (New York, Cambridge University Press, 1996) pp. 190.

5) J. Pilling and N. Ridley: Superplasticity in crystalline solids, (London, The institute of Metals, 1989).

6) R. Kaibyshev, I. Kazakulov, D. Gromov, F. Musin, D. R. Lesuer and T. G. Nieh: Scr. Mater. 44 (2001) 2411-2417.

7) K. Kannan, C. H. Jonson and C. H. Hamilton: Metall. Mater. Trans. 29A (1998) 1211-1220.

8) R. Verma, A. K. Ghosh, S. Kim and C. Kim: Mater. Sci. Eng. A191 (1995) 143-150.

9) O. A. Kaibyshev: Superplasticity of Alloys, Intermetallides, and Ceramics, (Berlin, Springer-Verlag, 1992) p. 317.

10) M. Watts, M. J. Stowell, B. L. Baikie and D. G. E. Owen: Metals Sci. 10 (1976) 189-206.

11) S. J. Hales and T. R. McNelley: Acta Metall. 36 (1988) 1229-1239.

12) Q. Liu, X. Huang, M. Yao and J. Yang: Acta Metal. Mater. 40 (1992) 1753-1762.

13) H. Yang, H. Miura and T. Sakai: Mater. Trans, JIM 37 (1996) 1379_ 1387.

14) J. Liu and D. J. Chakrabarti: Acta Mater. 44 (1996) 4647-4661.

15) T. Sakai, H. Yang and H. Miura: Mater. Sci. Eng. A234-236 (1997) $857-860$.

16) T. R. McNelley and M. E. McMahon: Metall. Mater. Trans. 28A (1997) 1879-1887.

17) T. G. Nieh, L. M. Hsiung, J. Wadsworth and R. Kaibyshev: Acta Mater. 
46 (1998) 2789-2800.

18) M. T. Perez-Prado, T. R. McNelley, O. A. Ruano and G. GonzalezDoncel: Metall. Mater. Trans. 29A (1998) 485-492.

19) T. R. McNelley, M. E. McMahon and M. T. Perez-Prado: Phil. Trans. R. Soc. Lond. A357 (1998) 1683-1705.

20) M. Eddahbi, T. R. McNelley and O. A. Ruano: Metall. Mater. Trans. 32A (2001) 1093-1102.

21) M. Biberger and W. Blum: Philos. Mag. A 65 (1992) 757-770.

22) R. Kaibyshev, T. Sakai, F. Musin, I. Nikulin and H. Miura: Scr. Mater.
45 (2001) 1373-1380.

23) T. Sakai, X. Yang and H. Miura: Light Metals 2000, ed. By J. Kazadi and J. Masounave (2000) 407-418.

24) M. G. Zelin, N. A. Krasilnikov, R. Z. Valiev, M. W. Grabski, H. S. Yang and A. K. Mukherjee: Acta Metall. Mater. 42 (1994) 119-126.

25) O. A. Kaibyshev, A. I. Pshenichniuk and V. V. Astanin: Acta Mater. 46 (1998) 4911-4916.

26) M. G. Zelin and A. K. Mukherjee: Philos. Mag. 68 (1993) 1183-1193. 